\title{
Effects of quarantine applied during the COVID-19 pandemic on mental health and quality of life in patients with multiple sclerosis and healthy controls
}

\author{
Emine Rabia Koc ${ }^{1} \cdot$ Aylin Bican Demir ${ }^{1} \cdot$ Ezgi Topaloglu $^{2} \cdot$ Omer Faruk Turan ${ }^{1} \cdot$ Guvan Ozkaya $^{3}$
}

Received: 26 October 2021 / Accepted: 14 January 2022 / Published online: 21 January 2022

(c) Fondazione Società Italiana di Neurologia 2022

\begin{abstract}
Background The coronavirus outbreak, which emerged in Wuhan, China, in late 2019 and spread to the world, has changed each of our lives.

Objective To investigate the effects of quarantine on depression, anxiety, sleep quality, fatigue, and SF-36 of multiple sclerosis (MS) patients during the COVID-19 outbreak and differences between healthy controls (HC).

Methods Eighty-six MS patients and $65 \mathrm{HC}$ patients were included in the study. Participants filled out the various scales through face-to-face interviews for mental health assessment from January 15 to February 15, 2021.

Results When both groups were compared in terms of BECK-D inventory $(p<0.001)$, BECK-A inventory $(p=0.010)$, and FS $(p<0.001)$, the patient group had significantly higher results. Physical functioning $(p<0.001)$, physical role limitation $(p=0.001)$, energy vitality rates $(p=0.010)$, and general health perception $(p<0.001)$ were higher in the HC group. When MS patients were divided according to EDSS scores, BECK-A $(p<0.001)$, BECK-D $(p=0.001)$, and PSQI $(p=0.006)$ scores of the patients with EDSS $>3$ were higher, while emotional role restriction rates $(p=0.006)$, energy and vitality $(p=0.018)$, and pain $(p=0.005)$ were significantly lower than those with EDSS $\leq 3$. When MS patients were divided into two groups as who had COVID-19 and who did not and compared SF-36 subscale scores, pain, $(p=0.049)$ and mental status $(p=0.030)$ were obtained significant differences in the two groups.

Conclusions Our study revealed that MS patients, who are more susceptible to the new 'normal' that emerged during the pandemic period, are among the priority groups that should be supported in terms of mental health as well as physical health.
\end{abstract}

Keywords COVID-19 · Quarantine · Multiple sclerosis · Mental health · Quality of life

\section{Introduction}

The epidemic of coronavirus, which emerged in Wuhan, China, in late 2019 and spread to the world, has changed our lives from top to bottom [1]. There have been many casualties in the world due to COVID-19. For this reason, governments have imposed some restrictions to prevent

Emine Rabia Koc

erabiakoc@yahoo.com

1 Department of Neurology, Faculty of Medicine, Uludag University, Bursa, Turkey

2 Department of Psychiatry, Faculty of Medicine, Uludag University, Bursa, Turkey

3 Department of Biostatistics, Faculty of Medicine, Uludag University, Bursa, Turkey the spread of the pandemic. Quarantine measures, which cover both infected and noninfected individuals, were also one of these. As of March 12, schools, restaurants, and cinemas were closed, national and international travel was suspended, and measures were expanded day-to-day in Turkey. The COVID-19 outbreak and the restrictions applied after it brought some psychological problems. Shigemura et al. suggest that fear of the unknown about the COVID-19 pandemic enhances individuals' anxiety levels and can cause several mental diseases [2]. Studies have also shown that chronic disease patients are more affected by these quarantine measures than healthy individuals. Ozamiz-Etxebarria et al. found that chronic disease patients had higher mental illness than healthy controls [3]. Maya Louvardi et al. investigated the effects of massive quarantine during COVID-19 on patients' mental health with chronic diseases. They found that while 
distress and somatization increased, anxiety and depression were not in this population. They also found different results among chronic disease groups. For example, patients with endocrine and cardiovascular disease had similar DSQ (Dimensional Symptom Questionnaire) findings as healthy individuals. In contrast, patients in the respiratory disease group had significantly higher scores in more than one domain (somatization and distress) than healthy individuals. Thus, evaluating the pandemic's psychological effects is essential for managing the symptoms in various patient groups during any crisis [4]. In this context, Costabile et al. reported that the depression detected in MS patients during the COVID-19 pandemic in the Italian society is higher than in healthy controls, and physical exercise is an important method to improve mental health and reduce the negative effects of disability on depression [5] Similarly, in the study of Carotenuto et al., it was shown that disability impacted depression during the COVID-19 pandemic, and recommending exercise for MS patients improves psychological health regardless of physical disability [6]. Another study emphasized that the pandemic might cause clinical and radiological worsening in MS patients through post-traumatic stress disorder, and therefore the importance of stress control and management [7]. Abbadessa et al. reported that the quality of life and mental health of people with MS were negatively affected by the pandemic and the necessity of supporting it with alternative methods like digital tools [8].

No study has been published comparing how MS patients and healthy individuals are affected by restrictions during the quarantine period in Turkey. In this study, we compared MS patients' and healthy controls' mental health during the COVID-19 pandemic and evaluated whether there was a difference between the two groups.

\section{Methods}

\section{Study designs}

This is a cross-sectional questionnaire study. The Beck Depression Inventory (BDI), the Beck Anxiety Inventory (BAI), the Fatigue Impact Scale (FIS), the Pittsburgh Sleep Quality Index (PSQI), and the Short Form of Quality of life (SF-36) were administered to the participants through faceto-face interviews in non-COVID-19 areas and single rooms, reserved for MS patients and their relatives or partners. The questionnaire forms were administered to individuals who agreed to participate in the study, and it took about thirty minutes to answer the questions. The study was approved by the Local Ethics Committee (Approval No: UU- 2021-1/29) and was conducted by the Declaration of Helsinki.

\section{Participants}

A total of 86 relapsing-remitting MS patients (57 women, 29 men) and 65 healthy controls (43 women, 22 men, and both groups age range 18 to 65 ) were admitted to the Neurology Clinic of Uludag University, Faculty of Medicine, from January 15, 2021, to February 15, 2021, were included in the study. The MS group was selected from patients whom MS specialists previously diagnosed, had regular outpatient follow-ups, and voluntarily accepted participating in the study. MS specialists evaluated patients' Expanded Disability Status Scale (EDSS) scores. Besides, patients who had an attack in the last 1 month, who were taking corticosteroid treatment, and who used regular medication for the last 6 months due to psychiatric problems or insomnia were also excluded from the study. The healthy control group was also selected from family members or partners of MS patients' who lived in the same environment as MS patients and agreed to participate in the study.

\section{Assessments}

Socio-demographic data of the participants include age, gender, marital status, living with family or a partner, having children, educational level, occupation, and smoking status.

The BDI consists of a self-reported instrument including 21-items that are used to assess characteristic symptoms of depression. Each question consists of four statements, each corresponding with a number from 0 to 3 . If a total score $\leq$ of 9 indicates "no depression," 10-16 indicates "mild depression," 17-23 indicates "moderate depression," and $\geq 24$ indicates "severe depression [9]."

BAI is a 21-question multiple-choice self-report inventory used to measure the severity of anxiety. Each answer is scored on a scale value of 0 (not at all) to 3 (severely), and higher total scores indicate more severe anxiety. The standardized cutoffs are: 8-15 indicate mild anxiety, 16-25 indicate moderate anxiety, and 26-63 indicate severe anxiety [10].

FFS is a 9-item questionnaire, and it was developed to measure fatigue in patients with neurological illnesses, particularly MS and systemic lupus erythematosus [11]. Answers are scored on a seven-point scale where " 1 " indicates strongly disagree, and " 7 " indicates strongly agree. The total score is calculated by arithmetic-mean, and a score of 4 or higher generally indicates severe fatigue [12].

PSQI is a self-report questionnaire that assesses the sleep quality of the participants over the last month. The scale consists of 19-items and seven components. The total score is between 0 and 21 , and a score of $\leq 5$ indicates "good sleepers" and $>5$ "bad sleepers [13]." 
The SF-36 is an instrument for evaluating health-related quality of life. It consists of eight sections which are physical functioning (PF), role physical (RP), bodily pain (BP), general health $(\mathrm{GH})$, vitality (VT), social functioning (SF), role emotional (RE), and mental health $(\mathrm{MH})$. Each section is scored in itself, and the higher total scores indicate better quality of life [14].

\section{Statistical analysis}

The data was examined by the Shapiro-Wilk test whether or not it presents normal distribution. The descriptive statistics were presented as mean \pm standard deviation, median (minimum-maximum), or frequency and percentage. Normally distributed data were compared with independent samples $t$-test. Mann-Whitney $U$ tests were used for non-normally distributed data. Categorical variables were compared using Pearson's chi-square test and Fisher's exact test between groups. $P<0.05$ was considered as significance levels. Statistical analyses were performed with IBM SPSS ver.23.0 (IBM Corp. Released 2015. IBM SPSS Statistics for Windows, Version 23.0. Armonk, NY: IBM Corp.).

\section{Results}

A total of 151 volunteers, 86 patients with MS and 65 healthy controls, participated in this study. The average age of the patient group was $38.14 \pm 11.01$, while it was
$38.00 \pm 12.5$ in the control group. There were $57(66.3 \%)$ females and $29(33.7 \%)$ males in the patient group, and 43 (66.2\%) females and $22(33.8 \%)$ males in the control group, with no statistical differences in terms of age $(p=0.942)$ and gender $(p=0.987)$.

There was no statistical difference in marital status in both groups $(p=0.693)$. When evaluated in terms of educational status, a statistically significant difference was found between the two groups $(p=0.045)$. While in the control group with healthy individuals, the educational status rate was $15.4 \%$, in the patient group, this rate was significantly lower at $4.7 \%$. Between these two groups, we also found a significant difference in terms of occupational groups $(p=0.019)$. The rate of homemakers in the control group was $12.3 \%$, yet this rate increases in the patient group with a percentage of $32.6 \%$. The civil servant's proportion is $30.8 \%$ in the healthy control group and is found this rate lower in the patient group with $16.3 \%$. The demographic data of the study groups are given in Table 1.

When both groups were compared in terms of BECK$\mathrm{D}$ inventory for depression $(p<0.001)$, BECK-A inventory for anxiety $(p=0.010)$, and FSS for fatigue $(p<0.001)$, the patient group had significantly higher results. Physical functioning ( $p<0.001)$, physical role limitation $(p=0.001)$, energy vitality $(p=0.010)$ rates, and general health perception $(p<0.001)$ were higher in the healthy control group. (Table 2).

When the patients were dichotomized into groups with a mild disability with EDSS $\leq 3.0$ or moderate disability
Table 1 Demographic and clinical features of participants

\begin{tabular}{lllll}
\hline & & Control $(n=65)$ & MS $(n=86)$ & $p$ \\
\hline Age & & $38.00 \pm 12.5$ & $38.14 \pm 11.01$ & 0.942 \\
Sex & Famale & $43(66.2 \%)$ & $57(66.3 \%)$ & 0.987 \\
Marital status & Married & $22(33.8 \%)$ & $29(33.7 \%)$ & \\
& Single & $41(63.1 \%)$ & $52(60.5 \%)$ & 0.693 \\
& Widower & $22(33.8 \%)$ & $27(31.4 \%)$ & \\
Educational Status & $1(1.5 \%)$ & $4(4.7 \%)$ & \\
& Divorced & $1(1.5 \%)$ & $3(3.5 \%)$ & \\
& Primary school & $6(9.2 \%)$ & $16(18.6 \%)$ & $\mathbf{0 . 0 4 5}$ \\
& Secondary school & $9(13.8 \%)$ & $7(8.1 \%)$ & \\
& High school & $14(21.5 \%)$ & $28(32.6 \%)$ & $31(36 \%)$ \\
Occupation & University & $26(40 \%)$ & $4(4.7 \%)$ & \\
& Postgraduate & $10(15.4 \%)$ & $28(32.6 \%)$ & $\mathbf{0 . 0 1 9}$ \\
& Housewife & $8(12.3 \%)$ & $4(4.7 \%)$ & \\
& Self-employed & $8(12.3 \%)$ & $11(12.8 \%)$ & \\
& Employee & $6(9.2 \%)$ & $14(16.3 \%)$ & \\
& Officer & $20(30.8 \%)$ & $11(12.8 \%)$ & \\
& Retired & $5(7.7 \%)$ & $6(7 \%)$ & \\
& Unemployed & $4(6.2 \%)$ & $12(14 \%)$ & \\
& Other & $14(21.5 \%)$ & & \\
& & &
\end{tabular}

All the statistically significant values have been highlighted in bold 
Table 2 Comparisons of questionnaire scores between MS patients and healthy controls (HCs)

\begin{tabular}{lcrr}
\hline & Control $(n=65)$ & MS $(n=86)$ & \multicolumn{1}{c}{$p$} \\
\hline BAI & $5(0-34)$ & $15(0-47)$ & $<\mathbf{0 . 0 0 1}$ \\
BDI & $8(0-26)$ & $13.5(0-43)$ & $\mathbf{0 . 0 1 0}$ \\
PSQI & $5(1-15)$ & $5(0-15)$ & 0.731 \\
FIS & $3.44(0-6.67)$ & $4.83(0.22-7)$ & $<\mathbf{0 . 0 0 1}$ \\
Physical functioning & $90(0-100)$ & $60(0-100)$ & $<\mathbf{0 . 0 0 1}$ \\
Physical role limitations & $100(0-100)$ & $50(0-100)$ & $\mathbf{0 . 0 0 1}$ \\
Emotional role limita- & $100(0-100)$ & $33.33(0-100)$ & 0.080 \\
$\quad$ tions & & & \\
Vitality & $55(5-100)$ & $45(0-100)$ & $\mathbf{0 . 0 1 0}$ \\
Emotional well-being & $64(0-96)$ & $56(8-96)$ & 0.055 \\
Social functioning & $62.5(0-100)$ & $62.5(0-100)$ & 0.650 \\
Pain & $80(0-100)$ & $67.5(20-100)$ & 0.128 \\
General health & $65(20-100)$ & $45(15-95)$ & $<\mathbf{0 . 0 0 1}$ \\
\hline
\end{tabular}

$B A I$, beck anxiety inventory; $B D I$, beck depression inventory; $P S Q I$, Pittsburgh sleep quality index; $F I S$, fatigue impact scale

All the statistically significant values have been highlighted in bold

with EDSS $>3$, in EDSS $>3$ group, the anxiety disorder rates according to the BECK-A scale $(p<0.001)$, depression according to the BECK-D scale $(p=0.001)$, sleep quality according to Pittsburg sleep quality index $(p=0.006)$ were found significantly higher, emotional role restriction rates $(p=0.006)$, energy and vitality $(p=0.018)$, and pain $(p=0.005)$ were significantly lower. When the patient group is divided into two groups in the COVID-19 pandemic as with and without acute attack, a significant difference was found in terms of fatigue $(p=0.040)$ and SF-36 sub-scales (physical function, $p=0.034$, and emotional role difficulties, $p=0.022$ ) between the two groups. Similarly, MS patients were divided into two groups as those who had COVID19 and those who did not, and compared SF-36 subscale scores, pain $(p=0.049)$, and mental status, $(p=0.030)$ were obtained significant differences in the two groups (Table 3 ).

\section{Discussions}

This study aimed to investigate the neuropsychological impact of the COVID-19 pandemic on MS patients and healthy controls. The main finding obtained from the study is that anxiety, depression, and fatigue are more common in MS patients than healthy controls during the pandemic period, and a difference was found between the two groups' SF-36 subscales scores.

During the pandemic, increasing some psychological problems is an expected condition. Several studies reported that the rate of anxiety had been increased in the general population [, 4, 4]. The anxiety level was higher in studies with chronic diseases and MS patients than in the healthy population, like in our study $[, 16,16]$. There could be many reasons for this situation; some of these are maybe prolonged pandemic duration, the time spent at home due to restrictions, the fear of falling ill more quickly than healthy individuals, the fear of losing relatives or losing their job. In addition to all these negative factors, in chronic diseases, such as MS, patients fear not reaching a doctor and not obtaining medication on time. All these factors may be the cause why MS patients have more anxiety than healthy controls.

On the other hand, Demir et al. found no significant increase in MS patients' anxiety symptoms [18]. This may be since the study was conducted within the first 3 months of the pandemic onset and that no patient had a COVID-19 infection. In the initial period of the pandemic, there was not enough information about MS itself and the drugs used in MS treatment about the risk of getting COVID-19 infection or how it will progress in MS patients after the infection [19]. This uncertain situation may also have caused anxiety in patients. Ahadi et al. have shown that anxiety developing in MS patients can cause disease exacerbation [20]. Therefore, it is crucial to deal with psychiatric problems, such as anxiety, in a timely and effective manner in MS patients.

While the restrictions imposed during the COVID-19 pandemic prevented the spread of the infection, they caused people to become more withdrawn. MS patients, especially those with physical disabilities, became more lonely over time as their already limited social facilities were further restricted. Besides, disruptions in physical and cognitive rehabilitation can cause patients to lose their previous gains. In our study, the higher scores of anxiety, depression, and sleep disorders in patients with an EDSS score over three may indicate the effect of disability on mental status. Previous studies have shown that the prevalence of depression in MS patients is higher than in the average population, regardless of the pandemic [21]. It is also maladaptive that MS patients, which are already prone to depression, develop strategies to cope with stress [22]. The aforementioned facilitating factors during the COVID-19 pandemic may explain why depression is higher in MS patients than in the healthy group, as shown in our and other studies [, 18, 18].

Fatigue is a common symptom affecting the quality of life in MS patients and is perceived by patients both physically and mentally [24]. There are many components in the pathophysiology of fatigue in MS, such as structural damage in the parenchyma, inflammatory processes, and maladaptive network [25]. Long-lasting fatigue symptoms during and after the infection have been reported in people with COVID-19 [26]. In our study, the lack of difference in fatigue between MS patients with and without COVID-19 infection suggests that the fatigue observed in MS is more related to the disease's pathophysiology. Also, our findings show that having 


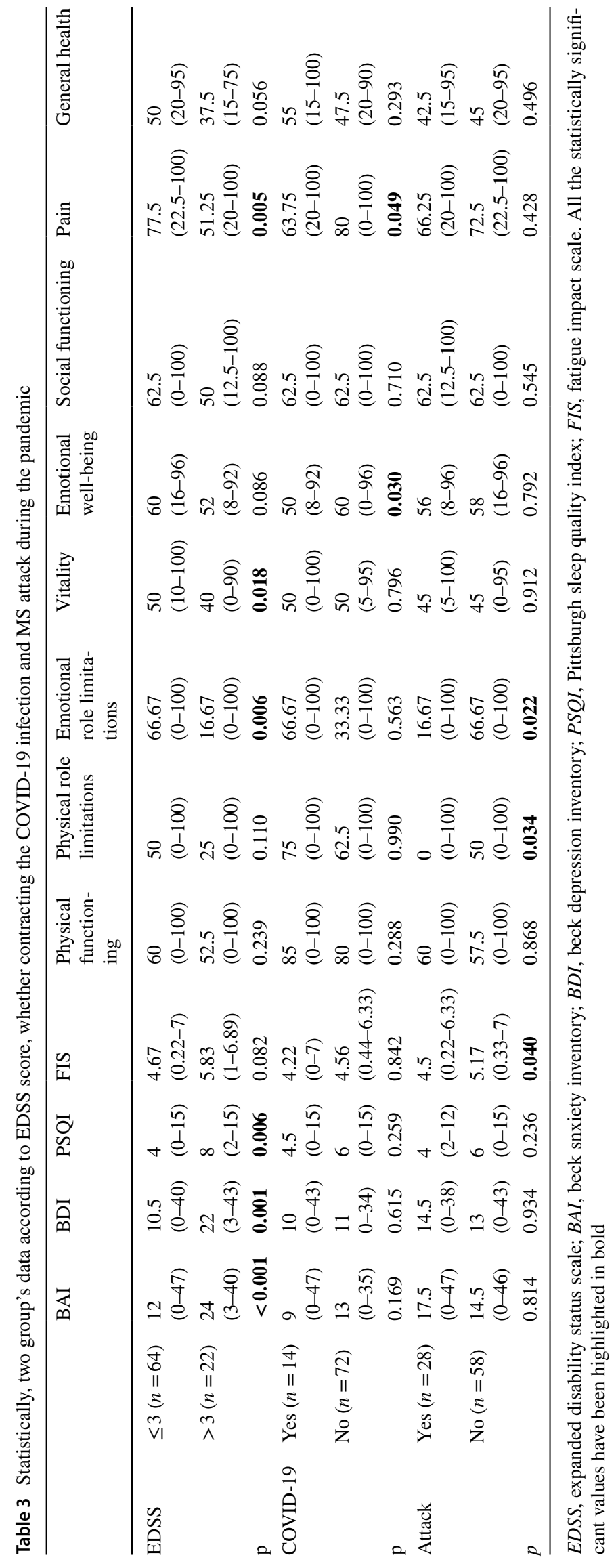


a COVID-19 infection does not affect sleep quality but that sleep quality deteriorates with increased disability.

Our study results indicate that MS patients had significantly worse health than healthy controls in physical function, role limitations-physical, vitality, and general health perceptions. On the other hand, there was no difference between MS patients and the healthy group in terms of emotional role, mental health, social functionality, and physical pain. This situation may be related to the disruption of MS patients' physical rehabilitation due to the restrictions applied during the pandemic period and the resulting deterioration of their quality of life. Besides, MS patients who contracted the COVID-19 were worse than the others in mental health and pain perception. The perception of physical and emotional role difficulties was worse in MS patients who had an attack during the pandemic than those who did not. For this reason, patients with high disability, COVID-19 infection, or MS attack during the pandemic period should be considered primarily to be included in psychological support programs.

In conclusion, MS patients' quality of life is mainly dependent on their mental and physical well-being. Since restrictions applied during the pandemic may lead to a lack of social input in MS patients, they should be supported mentally and cognitively. Online group activities can be organized with patient groups for patients to socialize. Also, routine visits and rehabilitation programs can be done with telemedicine to protect patients' physical and mental gains and increase.

Acknowledgements We appreciate the patients for cooperating and participating in the study.

\section{Declarations}

Ethics approval The study was approved by the Local Ethics Committee (approval no: UU- 2021-1/29) and was conducted by the Declaration of Helsinki.

Consent to participate Informed consent was obtained from all individual participants included in the study.

Conflict of interest The authors declare no competing interests.

\section{References}

1. URL. https://www.who.int/docs/default-source/coronaviruse/situa tion-reports/20200121-sitrep-1-2019-ncov.pdf?sfvrsn=20a99 c10_4. Accessed 17 Jul 2020

2. Shigemura J, Ursano RJ, Morganstein JC et al (2020) Public responses to the novel 2019 coronavirus (2019-nCoV) in Japan: mental health consequences and target populations. Psychiatry Clin Neurosci 74:281-282

3. Ozamiz-Etxebarria N, Dosil-Santamaria M, Picaza-Gorrochategui M, Idoiaga-Mondragon N (2020) Stress, anxiety, and depression levels in the initial stage of the COVID-19 outbreak in a population sample in the northern Spain. Cad Saude Publica 36:1-9. https://doi.org/10.1590/0102-311X00054020

4. Wang C, Pan R, Wan X et al (2020) Immediate psychological responses and associated factors during the initial stage of the 2019 coronavirus disease (COVID-19) epidemic among the general population in China. Int J Environ Res Public Health 17:1729

5. Costabile T, Carotenuto A, Lavorgna L et al (2021) COVID-19 pandemic and mental distress in multiple sclerosis: 1mplications for clinical management. Eur J Neurol 28:3375-3383

6. Carotenuto A, Scandurra C, Costabile T et al (2021) Physical exercise moderates the effects of disability on depression in people with multiple sclerosis during the COVID-19 outbreak. J Clin Med 10:1234

7. Bonavita S, Sparaco M, Russo A et al (2021) Perceived stress and social support in a large population of people with multiple sclerosis recruited online through the COVID-19 pandemic. Eur J Neurol 28:3396-3402

8. Abbadessa G, Lavorgna L, Trojsi F et al (2021) Understanding and managing the impact of the Covid-19 pandemic and lockdown on patients with multiple sclerosis. Expert Rev Neurother 21:731-743

9. Aydemir O, Guvenir T, Kuey LKS (1997) Reliability and validity of the Turkish version of hospital anxiety and depression scale. Turk J Psychiatry 8:280-287

10. Beck B, Epstein S (1988) An inventory for measuring clinical anxiety: psychometric properties. J Consult Clin Psychol 56:893-897

11. Krupp LB, LaRocca NG, Muir-Nash JSA (1989) The fatigue severity scale. Application to patients with multiple sclerosis and systemic lupus erythematosus. Arch Neurol 46:1121-1123

12. Anton HA, Miller WC, Townson AF (2008) Measuring fatigue in persons with spinal cord injury. Arch Phys Med Rehabil 89:538-542

13. Buysse DJ, Reynolds CF, Monk TH, Berman SR, Kupfer DJ et al (1989) The Pittsburgh Sleep Quality Index: a new instrument for psychiatric practice and research. Psychiatry Res 28:193-213

14. Lins L, Carvalho FM (2016) SF-36 total score as a single measure of health-related quality of life: scoping review. SAGE Open Med 4:205031211667172. https://doi.org/10.1177/2050312116671725

15. Luo M, Guo L, Yu M, Jiang W, Wang H (2020) The psychological and mental impact of coronavirus disease 2019 (COVID-19) on medical staff and general public - a systematic review and metaanalysis. Psychiatry Res 291:113190

16. Capuano R, Altieri M, Bisecco A et al (2021) Psychological consequences of COVID-19 pandemic in Italian MS patients: signs of resilience? J Neurol 268:743-750. https://doi.org/10.1007/ s00415-020-10099-9

17. Stojanov A, Malobabic M, Milosevic V et al (2020) Psychological status of patients with relapsing-remitting multiple sclerosis during coronavirus disease-2019 outbreak. Mult Scler Relat Disord 45:102407. https://doi.org/10.1016/j.msard.2020.102407

18. Demir CF, Bilek F, Balgetir F (2020) Neuropsychiatric changes during the COVID-19 pandemic in multiple sclerosis patients. Arq Neuropsiquiatr 78:570-575. https://doi.org/10.1590/0004-282x2 0200122

19. Giovannoni G, Hawkes C, Lechner-Scott J et al (2020) The COVID-19 pandemic and the use of MS disease-modifying therapies. Mult Scler Relat Disord 39:102073

20. Ahadi MS, Sahraian MA, Rezaeimanesh N, Moghadasi AN (2020) Psychiatric advice during COVID-19 pandemic for patients with multiple sclerosis. Iran J Psychiatry Behav Sci 14:. https://doi.org/ 10.5812/ijpbs. 103243

21. Feinstein A, Magalhaes S, Richard JF et al (2014) The link between multiple sclerosis and depression. Nat Rev Neurol 10:507-517 
22. Amato MP, Prestipino EBA (2019) Identifying risk factors for cognitive issues in multiple sclerosis. Expert Rev Neurother 19:333-347

23. Motolese F, Rossi M, Albergo G et al (2020) The psychological 1mpact of COVID-19 pandemic on people with multiple sclerosis. Front Neurol 11:580507. https://doi.org/10.3389/fneur.2020. 580507

24. Capone F, Motolese F, Falato E, et al (2020) The potential role of neurophysiology in the management of multiple sclerosis-related fatigue. Front Neurol 11:251

25. Manjaly ZM, Harrison NA, Critchley HD et al (2019) Pathophysiological and cognitive mechanisms of fatigue in multiple sclerosis. J Neurol Neurosurg Psychiatry 90:642-651
26. Carfî A, Bernabei R, Landi F (2020) Persistent symptoms in patients after acute COVID-19. JAMA - J Am Med Assoc 324:603-605

Publisher's note Springer Nature remains neutral with regard to jurisdictional claims in published maps and institutional affiliations. 